\title{
"Parlo assim para facilitar": la lingua italiana nelle Novelas paulistanas e nei Contos de Belazarte Maria Caterina Pincherle
}

\begin{abstract}
L'analisi linguistica dei racconti di Mário de Andrade e Alcântara Machado incentrati sugli immigrati italiani a San Paolo rivela un uso sorprendentemente variegato delle interferenze linguistiche dell'italiano sul portoghese, urilizzate come risorsa non solo in senso realistico, ma anche e sopracturto per creare effetti stilistici inconsueti.

PAROLE CHIAVE: italiano; portoghese; interferenze linguistiche; Mário de Andrade; Alcântara Machado; stilistica.
\end{abstract}

[...] Então os transatlânticos trouxeram da Europa outras raças aventureiras. Entre elas uma alegre que pisou na terra paulista cantando e na terra brotou e se alastrou como aquela planta também imigrante que há duzentos anos veio fundar a riqueza brasileira.

Do consórcio da gente imigrante com o ambiente, do consórcio da gente imigrante com a indígena nasceram os novos mamalucos.'

Così un quatrocentāo di tutto rispetto come Antônio de Alcântara Machado descrive negli anni Venti l'arrivo degli italiani in Brasile: una tappa naturale della storia del paese.

Il paragone stabilito dall'autore è interessante: la comunità degli immigrati si impianta e attecchisce nel Nuovo Mondo proprio come, due secoli prima, aveva fatro il caffe, quel prodotto nel quale ormai si identificava, inconfondibilmente e quasi per antonomasia, lo sviluppo del Paese. Nessuno dei due termini del paragone è nominato esplicitamente; attraverso una strategia del sottinteso ci viene presentata

1. Antônio de Alcânrara Machado, "Arrigo de fundo", pref. a Brás, Bexiga e Barra Funda, ora in Novelas Paulistanas, Rio de Janeiro, Garnier, 1994, p. 52. 
una sciarada, la cui unica soluzione possibile è l'inevitabile accostamento tra una coltivazione di grande successo e una popolazione dalle grandi promesse. Insieme, quindi, un clima di lieve mistero e di familiarità.

Tale commistione si ritrova in tutta la raccolta Brás, Bexiga e Barra Funda (1927), e risponde perfettamente alla dialettica distanza/avvicinamento che caratterizza il rapporto dell'autore con la materia narrata. In maniera potenziata, lo stesso trattamento viene riservato poco più tardi da Mário de Andrade ai personaggi e ai fatti dei suoi Contos de Belazarte, che proprio ad Alcântara Machado saranno dedicati.

Luso della lingua italiana nei racconti dei due autori risponde ad un trattamento analogo. Al contempo estranea ma comprensibile, essa si insinua nel tessuto della narrazione allo stesso modo in cui gli immigrati stavano penerrando nel tessuro urbano: a volte distinguendosi dal contesto in maniera evidente, altre volte con interazioni dalle sfumature sottili e variegate. Vorrei mostrare qui come la presenza dell'idioma straniero, lungi dal costituire un mero elemento decorativo o un semplice appoggio realistico per la creazione di tipi e ambienti, costituisca una risorsa nuova a cui i due autori attingono traendone effetti inconsueti, venendo quindi a rappresentare un elemento stilistico a pieno titolo.

Nell'analisi linguistica ravvicinata, il confronto tra i due autori potrà forse inoltre mostrarsi efficiente per uscire da una impasse critica, costituita più che altro da argomentazioni impressionistiche, talvolta contraddittorie, sulla maggior o minor "simpatia" dell'uno o dell'altro autore nei confronti della popolazione immigrata, in valutazioni di carattere e[ste]tico basate su una fusione approssimativa di aspetti emotivi e sociologici. Ad esempio, Mario Carelli e Giorgio Marotti, nelle loro panoramiche sui personaggi italiani nella letteratura brasiliana, hanno prediletto Alcântara Machado ritenendolo affetrivamente più partecipe al mondo degli immigrati ${ }^{2}$, mentre

2. Nel suo acclamato studio sugli italiani (persone reali e personaggi letrerari) a San Paolo negli anni '20, Carelli giudicava molto severamente la comparsa dell'atrributo carcamano in un dialogo di Belazarte il pesante epireto avrebbe rivelato il disagio (mal-estar) dello stesso Mário de Andrade nei confronti degli stranieri. Tutravia, lo stesso vocabolo pronunciato da un personaggio di Alcântara Machado veniva considerato una semplice registrazione del pregiudizio comune negli strati più conservatori dell'alta società di cui il racconto narra. In 
Alfredo Bosi sosciene il contrario ${ }^{3}$, e Rubens Ricupero si limita ad affermare una superiorità di Alcântara Machado come ritrattista degli italiani per una sorta di primato quantitativo ${ }^{4}$. Neanche l'analisi contenutistica sembra apportare elementi decisivi al riguardo - sempre che sia costruttiva una discussione di questo tenore - dato che un rapido confronto tra le due raccolte rivela notevoli tratti di similarità nella trama $\mathrm{e}$ nella caratterizzazione dei personaggi: delitti passionali; fanciulle che lasciano il devoro pretendente italiano per uno più ricco o più avventuroso; giovani figli di immigrati, nati in Brasile, fanaticamente pervasi di patriottismo per il paese d'adozione; imprenditori arrivisti che antepongono gli interessi alle relazioni interpersonali.

Fatte salve le ovvie differenze stilistiche - ellitrica e scattante la prosa di Alcântara Machado; autoriflessivo e disteso il procedere di Mário de Andrade - un confronto interessante potrebbe partire dal piano dell'organizzazione strutturale e linguistica dei testi.

altre parole, Alcântara Machado non avrebbe aderito alla visione xenofoba dei suoi personaggi, mentre Mário de Andrade, in certa misura, sì. La stessa inspiegata disparità di valutazione si ritrova per quanto riguarda la rappresentazione dei diferti degli iraliani (violenza, arrivismo, arroganza, mancanza di scrupoli), indice, secondo lo studioso, di severa critica da parte di Mário de Andrade ma non da parte di Alcântara Machado (Carcamanos e comendadores. Os italianos em Säo Paulo da realidade à fiç̣āo. 1919-1930. Árica: São Paulo, 1985, passim). Analogo, e altretranto poco giustificato, il giudizio di Marorti: "[Mário de Andrade] si avvicina al mondo della gente semplice, gli stessi personaggi che animano le Novelas Paulistanas di Alcântara Machado, conservando però un tono disincantato e critico, un linguaggio ironico e privo di simpatia per le figure che crea" (Il personaggio dell'italiano nel romanzo brasiliano dell"800 e '900. Roma: Bulzoni, 1978, p. 28).

3. “[...] há muito de divertissement nas páginas do paulistano [Alcântara Machado]. Nelas, uma análise ideoestilística mais rigorosa não conşata nenhuma identificaçăo coerente com o imigrante, "pitoresco" no máximo, patético porque criança (o conto célebre do Gaetaninho), mas, em geral, ambicioso, petulante, quando capaz de competir com as famílias tradicionais em declínio. O populismo literário é ambíguo: sentimental, mas intimamente distante. [...] é sensivel, a uma leitura critica dos contos, esse fatal olhar de fora os novos bairtos operários e de classe média a crescerem e consolidarem uma nova São Paulo [...]" (A. Bosi, História concisa da literatura brasileira. São Paulo: Cultrix, 1990, p. 423).

4. Nella sua interessante introduzione, parzialmente aurobiografica, all'edizione iraliana di Brás, Bexiga e Barra Funda, Ricupero afferma che in Mário de Andrade l'immigrato sarebbe stato solo 'personaggio episodico" mentre in Alcântara Machado avrebbe costituito "la trama stessa, il nucleo centrale, la materia esclusiva dell'opera letteraria" (Nosizie di Säo Paulo. Milano: Scheiwiller, 1981, p. 11). 
Un primo dato è il diverso carattere di familiarità che i due autori hanno voluto attribuire alle loro raccolte, introducendo un filtro diegetico "amico" nella narrazione: più obiettivo e distaccato in Alcântara Machado, che si finge "professionista" nelle vesti del cronista portavoce della comunità italo-paulista ("Brás, Bexiga e Barra Funda é o órgão dos ítalo-brasileiros de São Paulo. [...] É um jornal. Mais nada.[...] Não comenta. Não discute. Não aprofunda."); più "caldo", dal punto di vista della letterarietà, in Mário de Andrade, che elegge un narratore di secondo grado, Belazarte, il quale gli presenta le vicende come storie vere e recenti in maniera del tutto colloquiale, come se esse appartenessero ad un mondo vicino sia a lui, sia a noi lettori. Belazarte riprende a volte gli stessi personaggi in racconti diversi, con la precauzione che si adotterebbe, nel parlato, contro il rischio di oblio o di confusione: "Você se lembra do João? Ara, se lembra! o padeiro que gostava da Rosa, aquela uma que casou com o mulato..."; "Você inda está lembrado da Teresinha? aquela uma que...", con un efficace gioco di deissi. Altre volte, l'autore viene direttamente chiamato in causa dal narratore nelle sue vesti reali ("Você é músico, e do conservatório grande lá da avenida São João, porisso há de se divertir com isso"), di nuovo all'insegna della familiarità. E fin dalla scelta del nome, Belazarte, si sfrutta, destituendola della caratteristica asprezza, la notorietà del furfantello della narrativa popolare Malasartes.

Nessuna di queste modalità, tuttavia, è legata in maniera esclusiva al mondo degli immigrati; esse lo rendono solo più "simpatico", per usare il termine impiegato da Marotti.

E invece sul piano della lingua che si rende palese il vincolo specifico con il contesto e l'oggetto della narrazione. L'accoglienza del vocabolo italiano nella prosa brasiliana (modernismo oblige: l'aggettivo brasiliano si impone in questa fase di sperimentazione letteraria nazionale) indica, già di per sé, la disposizione a trasformare il proprio medium, a plasmarlo per renderlo arto a riprodurre una realtà nuova, utilizzando una risorsa propria di quella stessa realtà: la mimesi in questo caso non consiste solo nella creazione di un ritratto verosimile a partire da un modello vivente, ma nell'uso del "materiale" pittorico prelevato dal modello stesso. 
Ciò che viene rilevato da entrambi gli autori è, in maniera specifica, il processo di interferenza tra le due lingue, come traccia della fase di adattamento degli stranieri. La resa realistica attraverso gli inserti italiani interessa, perciò, non tanto per il grado di realismo raggiunto (tratti di una lingua o di un dialetto italiano impeccabili) quanto per l'intenzione: da un punto di vista strettamente tecnico, un testo che avesse voluto riprodurre in maniera rigorosa il parlato degli italiani nella fase del primo adattamento forse sarebbe risultato poco comprensibile ai lettori brasiliani, quindi poco interessante e pertanto, al limite, inutile.

Quello che più importa è rendere non solo fruibile ma godibile ai lettori il pastiche, facendone uno strumento di caratterizzazione psicologica o sociologica al di là del macchiettismo. Non viene cercato l'effetto di ridicolo attraverso la caricatura; né, d'altro canto, si ambisce alla registrazione meccanica e scientifica di una modalità verbale: quella che viene promossa è l'espressività in sé, in uno stile che si potrebbe definire realismo espressionistico - quando, cioè, l'autore calca sul segno in modo particolare, utilizzandone l'effetro di distorsione. Può quindi capitare che, nell'intento di ri-produrre (produrre di nuovo, e non solo replicare) l'adattamento fonetico, lessicale e fraseologico degli italo-paulistani, l'autore brasiliano infili delle espressioni assolutamente inverosimili in bocca al suo personaggio: questi adattamenti, pur scorretti dal punto di vista prettamente linguistico, risultano tuttavia pienamente rispondenti all'intento stilistico, e, soprattutto, riconoscibili a un lettore di lingua portogheses. Detto per inciso, possono essere talvolta proprio questi errori a caricare il testo di

5. In un saggio sulla lingua di Goldoni, Gianfranco Folena notava come, nell'intento di imitare un francese che si sforava di parlare icaliano, il commediografo usasse un franco-italiano grammaricalmente incredibile in bocca a un francese che italianizzi", con una "fenomenologia dell' interferenza linguistica capovolta rispetto a quella probabile". Tuttavia questa, che cra proprio "la prospertiva del pubblico che ascolta[va]" e che riconosce[va] specularmente gli sproposiri abiruali di un iraliano quando cerca di parlare il francese senza conoscerlo", aveva molta più efficacia dal pun to di vista dello spettacolo. "Se pure orripilante, it procedimento è rivolto a ottenere un facile efferto di comicità verbale: il processo contrario, reale, avrebbe lasciato freddo il pubblico, almeno quello che non conoscesse bene il francese". G. Folena, "Il francese di Goldoni", in Litaliano in Europa. Esperienze linguistiche del Settecento, Torino: Einaudi 1983, p. 371. 
un valore aggiunto, di un effetro o una sfumatura che l'interferenza verosimile non avrebbe avuto. Definirò allora questi casi come "errori funzionali"6.

La selezione di esempi che segue ambisce a mostrare come gli interventi della lingua italiana nel parlato dei personaggi stranieri porti, nei racconti di Alcântara Machado e Mário de Andrade, ad una notevole diversità di effetti a partire da un'unica variabile, quella dell'interferenza della lingua madre nella lingua seconda.

\section{Il collage $\mathbf{c}$ i suoi lettori}

Il grado più semplice di interferenza testuale della lingua straniera è il collage ovvero l'inserzione di un brano in italiano senza adattamenti, all'interno del contesto linguistico portoghese. Tuttavia la semplicità di questa tecnica, se analizzata dal punto di vista del lettore, può rivelare una efficacia espressiva notevole.

Il racconto "Nacionalidade" di Alcântara Machado si apre con il barbiere "Tranquillo Zampinetti da rua do Gasômetro n ${ }^{\circ} 224-B^{\prime}$ " che legge ad alta voce un comunicato di guerra dal Fanfulla, il diffusissimo giornale degli italiani a São Paulo.

6. Chiamo errore funzionale un ripo di errore involontario, che - proprio in quanto errore, fuori dallo schema di prevedibilità della grammatica normativa - può arrivare a "funzionare" meglio del testo corretto, apportando un insperato carattere di originalità all'interno del linguaggio usato. Anni fa, ad una rassegna cinematografica a Rio de Janeiro, Alain Robbe-Grillet descrisse le sue prime esperienze come regista, ed i vari "errori" di montaggio che gli segnalava, scandalizzara, la script. Fece al pubblico l'esempio di una scena in cui un uomo si dirige verso una catredra e vi si siede. Per non dover mostrare tutta la lunga sequenza, il regista avrebbe tagliato la scena della camminata, per mostrare immediatamente dopo il personaggio seduto. Ma in tal modo, secondo la script, lo spettatore avrebbe avuto l'impressione che il personaggio stesse andando verso un posto in cui si trovava già. Il regista norò che, efferrivamente, in qualche modo, è proprio così: a chiunque si diriga verso una meta capita di immaginare di esserci già: e pur non essendo stato quello l'effetto voluto originariamente, l'accogliere il grossolano errore di sintassi del montaggio avrebbe permesso di mostrare la percezione soggetriva del protagonista, trasformando una semplice scena descritriva in una molto più ricca di suggestione.

7. Di "collage" riguardo alla prosa di Alcânrara Machado parla, anche se in un'accezione diversa, Vera Chalmers, che usa l'espressione "colagem cinérica do movimento social" ("Virado à paulista", in Os pobres na literatura brasileira, a c. di Roberto Schwarz. São Paulo, Brasiliense, 1983, pp. 136-139) 
La fulminante investita dei nostri bravi bersaglieri ha ridotto le posizione nemiche in un vero amazzo di rovine. Nel campo di battaglia sono restati circa cento e novanta nemici. [...]

Ora, è chiaro che un intero paragrafo di questo tenore, per lo meno in parte oscuro ai lettori che non abbiano discrete nozioni di italiano, non può certo funzionare in maniera significativa sul piano del contenuto. Viceversa, sul piano della suggestione visiva e auditiva esso può avere, proprio per la sua incomprensibilità, una immensa efficacia. Nella scena dal barbiere, questa sorta di colonna sonora straniera, di cui il brano offre gli inutili sottotitoli in lingua originale, immerge infatti totalmente il lettore in un altro mondo, sebbene fin dall'incipit gli fossero state date le coordinate spaziali, con tanto di indirizzo preciso. Una spiazzante icona sinestesica.

Inoltre, sul piano emotivo e su quello dell'immaginazione, chiunque abbia la pazienza di scorrere tutto il semi-incomprensibile brano vedrà intensificata, insieme alla propria frustrazione, la sensazione di arbitrarietà del "puro entusiasmo" di un personaggio come Zampinetti, che giubila per conquiste coloniali di una patria che si trova a migliaia di miglia da lui e cerca di contagiare nella sua euforia clienti che non la possono affatto condividere. Le stesse notizie, del resto, pur scritte in italiano, sono stampate in Brasile, ben lontano dai luoghi interessati (sia da quelli dove avvengono i fatti, il Nordafrica, sia da quelli dove i fatti potrebbero risultare rilevanti, l'Italia). Altrettanto straniante deve suonare il ritornello "Tripoli sarà italiana, sarà italiana a rombo di cannone!", con le esclamazioni correlate, pronunciate brandendo "a navalha como uma espada" di fronte a "fregueses assustados"... Ma per un momento, il tempo di leggere il trafiletto in italiano, lo "straniero" nel racconto sarà il lettore brasiliano, che potrà, per empatia, immedesimarsi specularmente negli immigrati in terra straniera.

Quanto all'ottica di un molto meno probabile lettore italiano, in questo caso, trattandosi di una riproduzione meccanica, le due imperfezioni di italiano nel brano del Fanfulla, "le posizione" e "amazzo di rovine" danno l'impressione di refusi poco verosimili, fornendo un chiaro indizio sull'origine straniera dello scrittorecopista (che non ha familiarità con le doppie, né con il plurale irregolare). Un caso diverso parrebbe invece essere quello dell'insulto rivolto, in questo stesso raccon- 
to, dal protagonista al figlio Lorenzo, che lo infastidisce perché non vuol parlare italiano: "- Stai attento que ti rompo la faccia, figlio d'un cane sozzaglione, che non sei altro!" Pur dubbia l'efficacia dell'insulto in sé (che si ritorce contro chi lo sta facendo), le espressioni colloquiali sono più che verosimili perfino nella loro variante dialettale ("sozzaglione"). E quel "que" sfuggito all'autore, invece di allontanare drasticamente la copia dal suo modello veridico, ha quasi l'effetto contrario, poiché rende in diretta un esempio del grado di penetrazione della lingua italiana nell'ambiente brasiliano: l'autore capta i suoni italiani (il nuovo sound della città) e li riproduce senza mediazioni secondo la sua fonetica. In quest'ottica, pare libresca, al confronto, la seconda forma corretta ("che") nell'esclamazione finale, frutto di un approccio con la lingua italiana passato attraverso la scrittura.

I dialoghi e i loro protagonisti

Interferenze linguistiche ben più complesse si trovano nei dialoghi tra personaggi italiani e brasiliani, in cui fanno la loro comparsa prestiti, adattamenti e calchi sia morfosintattici sia lessicali e semantici. Dialoghi bilingui di questo tipo rispondono maggiormente a quella dialettica distanza/avvicinamento cui si accennava.

Sia i personaggi sia la lingua appaiono, nei racconti di Alcântara Machado e di Mário de Andrade, sufficientemente inseriti nel quotidiano dei paulisti da rendersi comprensibili, nel senso più ampio e più etimologico del termine: entrambi stanno di fatto passando attraverso un processo di incorporazione nel testo/tessuto della città in espansione. Se la riproduzione della lingua ibrida parlata dagli immigrati marca la loro distanza rispetto al contesto, essa è, al tempo stesso, indice palpabile del loro processo di inserimento.

In "Menina de ôlho no fundo", di Mário de Andrade, mentre la protagonista Dolores incarna il desiderio, da parte della seconda generazione di immigrati, di sentirsi non solo integrata nella nuova società, ma di essere anche pienamente definita come brasiliana (e vale la pena rileggere gli spassosi confronti fra l'Italia e il Brasile fatti dalla ragazzina), il personaggio del Maestro Marchese è l'unico, tra 
tutti quelli di Belazarte, ad essere presentato con tratti apertamente caricaturali, che in qualche modo lo avvicinano al grottesco Venceslau Pietro Pietra di Macunatma: "era Maestro uma ova, [...] esfregava rabecão num barzinho de Gênova, não sei [...] virou rei da música do Brás. [...] já sabe: o Maestro Marchese já de brilhantão no dêdo e quatro marchesinhos com bastante macarrāo na barriga lá em casa”, dove compare l'elemento più trito del luogo comune nazionale, la pasta...

La trama, in breve, è questa: invaghitasi di Gomes, il suo professore di violino, la bella Dolores mette in giro infondate quanto compromettenti voci sul loro conto, con l'unico risultato che il Maestro Marchese, diretrore della scuola, costringe l'amato Gomes a dimettersi.

Coerentemente con l'identità dei personaggi, viene rappresentata la loro competenza linguistica: se il portoghese di Dolores non risente affatro della sua nazionalità di origine, quella del Maestro riflette in maniera sorprendente la dinamica del processo di integrazione e ascesa sociale. Tuttavia, mentre i tratti caricaturali riguardano essenzialmente la notazione caratteriale e di classe - l'esibizionismo pacchiano e arrogante del nouveau-riche -, a livello linguistico e stilistico l'effetto è più sottile. La strategia discorsiva e $\mathrm{i}$ contenuti delle battute del Maestro risentono infatri sempre della sua indole ipocrita, laddove il piano strettamente linguistico, quello delle interferenze italiano-portoghese, è sfrurtato in modo del turto indipendente dall'aspetro più immediatamente caricaturale.

È all'interno del dialogo fra il Maestro e Gomes che si evidenziano questi fenomeni. L'untuosità del nostro parvenu appare infatti proprio nelle battute rivolte al suo dipendente, che al termine del colloquio darà le dimissioni. Il Maestro comincia con l'insultare il violinista come donnaiolo che ha scelto il posto sbagliato per i suoi "scherzi con moças"; alle proteste di lui, cambia registro, blandendolo e trattandolo come "honestissimo", fa appello alla sua pietà di fronte ad un padre di famiglia, e lo fa accomodare; visto che il suo interlocutore non cede, cerca di risvegliare in lui uno sbilanciatissimo sentimento di giustizia (verso il quartiere e interamente a scapito suo, indebitamente sacrificato): "Bisogna dare una satisfazione a tutto il Brás!" Il giovane, sfinito dall'altalena di insulti e lusinghe, capitola. 
In tutti i discorsi del maestro, i tratti di interferenza dell' italiano fungono invece da sensibilissimo termometro dello stato emotivo del personaggio, con un effetto, come si accennava, molto più sottile che quello macchiettistico (turt'al più, si potrà dire che le pretese del parvenu risaltano ancor di più nei tratti in cui emerge con chiarezza - attraverso il linguaggio appunto - la sua origine, sicuramente umile come quella di qualsiasi emigrante. Ma si tratta, per così dire, di un efferto collaterale).

Un primo segnale in questo senso lo troviamo nell'autocompiacimento con cui il Maestro presenta per la prima volta il suo isrituro alla madre della potenziale allieva Dolores, quasi parlando fra sé e sé nella considerazione finale pronunciata pressoché integralmente in italiano - considerazione autodiretta, come si addice al sentimento di orgoglio che la anima:

Por isso o Maestro falou prá mai da mocinha que a sinhora vai vedere que num estantinho sua filha fica una artista, lo giuro! Seu Gomes é un professore molto bon, ah questo!... proprio la minha scuola! ${ }^{8}$

$\mathrm{Ma}$, analizzando il dialogo con Gomes, apparirà la progressione dinamica di questo legame tra emozioni e lingua:

- Bom dia, s'accomodi. Professore, mandei chamar o signore por causa dum assunto molto serio! Il Giacomo é un stabilimento serio! Qui non si fa scherzi com moças, signor professore! Si lei aveva l'intenzione di namorare careceva de andare noutro...

$-(\ldots)$

- Ma non dzangare! Non dzan-ga-te, signor professore! Non cé mica male in quello que eu disse! Sei molto bene que lei é honestissimo mache posso fare, io! Todos falam! S'accomodi, per favore!

$-(\ldots)$

- Ma non dzangate, signor fessore!... Stó falando sul serio! Sono un povero uomo con quatro figlioli in casa, si! signor professore, che belleza de criancinhas! Non posso expulsare questa ragazza Bermudes sinon m'isculhamba tutta la vida! Sono inrovinato, Dio santo! nāo posso mandare la ragazza s'imbora! é o non é!...

$-(\ldots)$

8. Mário de Andrade, Os conzos de Belazarte. São Paulo: Itatiaia, 1980, p. 64. 
- Ma, signore professore, non basta! Bermudes stá una fera! e io ho paúra dum scandalo!... Bisogna dare una satisfazione a tutto il Brás!...

$-(\ldots)$

- Bravo! Si vede che lei é um bravo moço! Sempre falei pra todos que lei é um bravo moço!

$-(\ldots)$

- Ah, ma o signore si esquece do dinheiro, isto nó! Mancano cinco dias ma il Giacomo paga tutte le mensalitá. Tante grazie, signor professore, tante grazie!... á rivederlo!?

In ogni battuta del Maestro Marchese sono presenti fortissimi tratti di interferenza e di ibridazione, al punto che si oscilla tra un portoghese fortemente italianizzato e, viceversa, un italiano fortemente portoghesizzato. Si verifica un continuo alternarsi di elementi lessicali, sintattici e fraseologici delle due lingue, in varie modalità: il trasferimento di morfemi da una lingua all'altra ("satisfazione", "mensalità"; verbi portoghesi con desinenze italiane: "arranjare", "tocare", "namorare", "careceva" "expulsare", "non dzangate", alcuni dei quali improbabili: "sono inrovinato"); l'inserimento di elementi lessicali italiani in strutture sintattiche esclusivamente portoghesi ("vai vedere", "pra la signorina tocare" e l'ibrido "mandare la ragazza s'imbora") o viceversa ("bisognava arranjare"); l'adattamento fonerico ("non dzangate", che riproduce graficamente la pronuncia italiana), spesso reso esclusivamente ai fini della lettura, attraverso la grafia ("sinhora" "qualque" "paúra").

Ognuna di queste modalità di interferenza è perfettamente attestabile nella fase iniziale dell'adattamento linguistico corrispondente al processo di integrazione sociale degli immigrati - nel momento, cioè, in cui questi si sforzano di parlare la lingua del paese di adozione, pur lasciandosi sfuggire di continuo elementi della propria lingua madre ${ }^{10}$.

Ma l'adozione di termini o locuzioni italiane da parte del Maestro non è distribuita in maniera regolare: come si diceva, essa è infatti direttamente propor-

9. Ibid., pp. 80-81.

10. In generale, per $\mathrm{i}$ tratti linguistici dell'italiano maggiormente soggetti a deformazioni al contatto con altre lingue, in situazioni di immigrazione, si è fatto riferimento a Gaetano Berruto, Sociolinguistica dell'italiano contemporaneo. Roma: La Nuova Italia Scientifica, 1989, pp. 179-186. 
zionale all'impatto emotivo che un certo argomento ha su di lui in quell'istante. Nei momenti in cui più vuol porre l'accento sul suo interesse e la sua reputazione (l'“onore" latino!), messa in gioco da un possibile scandalo, il Maestro passa francamente all'italiano:

Il Giacomo é un stabilimento serio! Qui non si fa scherzi com moças, signor professore!, e lo stesso avviene quando emergono la sua paúra e, simmetricamente, a pericolo scongiurato, il suo sollievo:

Sono un povero uomo con quatro figlioli in casa (...). Sono inrovinato, Dio santo!

Bravo! Si vede che lei è um bravo moço!

(...) ma il Giacomo paga tutte le mensalitá. Tante grazie, signor professore, tante grazie!... A rivederlo!

$\mathrm{Da}$ notare che se alcune di queste frasi sono perfettamente comprensibili, pur comprendendo un'alta percentuale di elementi dell'italiano, altre sono criptiche per un brasiliano per il quale termini come 'scherzi' e stabilimento' sono piuttosto distanti dagli equivalenti portoghesi.

Ben al di là della tentazione macchiettistica che pure connotava inizialmente la costruzione di questo personaggio, la sua parlata riflette un intento molto più realistico, raggiungendo un effetto di introspezione psicologica inconsuero e estremamente profondo. Nel regolare l'ingresso della lingua italiana secondo il fattore emotivo, l'autore si avvicina alla verità fenomenologica dei rapporti interpersonali tra emigranti e brasiliani in una maniera che sfiora i limiti del paradosso: se la pressione emotiva subita dal Maestro può a volte essere tale da prendere il sopravvento sulla piana esigenza di comunicare (rendendo incomprensibile quanto sta dicendo ai suoi interlocutori brasiliani), la volontà da parte dell'autore di restituire questo meccanismo può rischiare di rendere le battute del suo personaggio incomprensibili ai lettori. Ma questo stesso effetto, simile a quello del "collage" di Alcântara Machado, faciliterà ancor di più una loro immedesimazione in questo genere di situazioni sociali e comunicative. 
Un altro dialogo mistilingue, ma con effetti ancora diversi, si trova in "A sociedade" di Alcântara Machado.

Il racconto si apre con la battuta "Minha filha não casa com filho de carcamano!" per concludersi immancabilmente con l'annuncio matrimoniale. Fra i due estremi, una proposta d'affari, efficacissimo catalizzatore che rovescia, in maniera istantanea, il giudizio del paulista sulla famiglia del giovane pretendente. Il carcamano in questione, "o italiano das batatas" si presenta al futuro consuocero come "il capitale" della eventuale nuova società, della quale il figlio dovrebbe diventare il direttore. Il colloquio tra i due padri di famiglia si svolge a casa del brasiliano, dove il Cav. Uff. Salvatore Melli dimostra con grande efficacia "as vantagens econômicas de sua proposta" Il dialogo, uno dei pochi in cui Alcântara Machado ricrea l'italo-portoghese degli immigrati, è ricchissimo di trovate:

\section{-O Doutor...}

-Eu não sou doutor, Senhor Melli.

-Parlo assim para facilitar. Non è para ofender. Primo o doutor pense bem. E poi me dê a sua resposta. Domani, dopo domani, na outra semana. Io resto à sua disposiçāo. Ma pense bem!

Renovou a proposta e repetiu os argumentos pró. [...] Constitulam uma sociedade. O conselheiro entrava com os terrenos. O Cav. Uff. com o capital. Arruavam os trinta alqueires e vendiam logo para os operários a fábrica. Lucro certo, mais que certo, garantidíssimo.

-É. Eu já pensei nisso. Mas sem o capital o senhor compreende é impossível...

-Per Bacco, doutor! Mas io tenho o capital. O capital sono io. Mais nada. E o lucro se divide no meio.

O capital acendeu um charuto. $O$ conselheiro coçou os joelhos disfarçando a emoção. A negra de broche serviu o café.

-Dopo o doutor me dá a resposta. Io só digo isto: pense bem.

O capital levantou-se. Deu dois passos. Parou. Meio embaraçado. Apontou para um quadro.

-Bonita pintura.

Pensou que fosse obra de italiano. Mas era de francês.

-Francese? Não é feio non. Serve.

Embatucou. Tinha qualquer cousa. [...] Decidiu-se.

-Ia dimenticando de dizer. O meu filho fará o gerente da sociedade... Sob a minha direçăo, si capisce. 
-Sei, sei. O seu filho?

-Si. O Adriano. O douror... mi pare... mi pare que conhece ele?

O silêncio do Conselheiro desviou os olhos do Cav. Uff. na direção da porta.

-Repito un'altra vez: o doutor pense bem.

O Isotta Fraschini esperava-o todo iluminado.

[...]

A outra proposta foi feita de fraque e veio seis meses depois."

Come sempre nei racconti di Alcântara Machado, la conclusione è stringata, e consiste nella semplice riproduzione grafica degli annunci matrimoniali, quasi, di nuovo, un collage-testimoniale. In una sorta di epilogo narrarivo, durante la festa di fidanzamento la madre di Adriano ricorda alla consuocera "os bons tempinhos em que lhe vendia cebolas e batatas, Olio di Lucca e bacalhau português, quase sempre fiado e até sem caderneta" ${ }^{12}$ : senza alcuna remora, viene rievocata la condizione umile delle due famiglie. La riconciliazione è completa, l'ordine degli affari e quello domestico si sono ricongiunti, a piena garanzia di un futuro radioso...

La trasformazione radicale si fa proprio attraverso quegli elementi su cui poggiava il pregiudizio: lo stesso senso degli affari, prima spregiativamente reputato come prerogativa del "ladro", viene ora ammirato come dote preziosa da emulare e concretizzare $^{13}$. Nel dialogo decisivo, $l^{\text {'s }}$ alterità" dell'italiano viene marcata proprio dall'uso della lingua spuria, e l'analisi delle interferenze dell'italiano sul portoghese rivela un effetto espressivo che non si riscontra in Mário de Andrade. Se in "Menina de ôlho no fundo" alcune frasi venivano pronunciate dall'immigrato in preda alle emozioni quasi prescindendo dalla loro comprensibilità, in questo caso i termini ita-

11. Brás, Bexiga e Barra Funda, cit., pp. 76-77. I corsivi rispettano l'edizione citara.

12. Ibid., p. 78.

13. Marotri nota come, in maniera generale, quello che accomuna tutti i personaggi italiani nella letreratura brasiliana è il fatto di appartenere "in un modo o nell'altro al mondo del lavoro, ovvero, [sono] persone che esercitano un'artività per poter vivere; e questo, che può parere ovvio se non addiritrura banale, ha la sua importanza in una letteratura come quella brasiliana, tutta piena di personaggi votati al dolce farniente, sempre inclini a evitare qualsiasi contatto con il mondo del lavoro" (Il personaggio..., cit., p. 8). Analogamente Mário Caval canti Proença rifletteva sull'antagonismo implicito fra Macunaima e il suo nemico Venceslau, incentrato sul fatto che questi incarnava to senso prático das coisas" (Roteiro de Macunaima. São Paulo: Anhembi, 1955, p. 28). 
liani sono ridotti al minimo, ma (a meno che non compaiano come meri riempitivi fraseologici, come "per Bacco" o "si capisce") la loro presenza è sempre strategica. E perfino la mancata verosimiglianza linguistica, che non si attesta quasi in Mário de Andrade, agisce in questo caso come errore funzionale, potenziando l'efficacia espressiva degli inserti italiani.

La contaminazione tra italiano e portoghese riprodotta in questo dialogo non è infatti per nulla attendibile sul piano realistico: il Cav. Uff. usa perfettamente forme grammaticali complesse, nient'affatto spontanee in uno straniero, come il congiuntivo esortativo ("me dê" invece del popolare "me dá", "pense bem"), il congiuntivo futuro ("quando quiser") o la forma continuativa ir+gerundio, che non hanno usi equivalenti in italiano. Segni, questi, di notevole dimestichezza con la lingua d'adozione, e che vengono peraltro confermati dalla spontaneità nell'uso di caratteristiche fraseologiche del parlato: la ripetizione della negazione "nāo é feio non" (pur con l'imitazione soltanto parziale della imperfetta pronuncia nasale), e addirittura il solecismo "o senhor, mi pare que conhece ele" invece di "que o conhece", sicuro prodotto del contagio con il portoghese parlato, e altrimenti impensabile in un italiano la cui forma standard "che lo conosce" avrebbe portato spontaneamente alla forma corretta in portoghese.

Tuttavia, come se improvvisamente questa solida base venisse a mancare, il Cav. Uff. si lascia sfuggire italianismi dei più banali, assolutamente gratuiti, dei quali è impossibile che non conosca l'equivalente in portoghese, data la loro frequenza nel parlato quotidiano: uno fra tutti, "io" per "eu". Altre volte, alterna nello stesso dialogo la forma italiana e quella portoghese: "parlo" per "falo" o "digo" (mentre poi usa "só digo isto"), "non" per "nāo"

In sostanza, sembra che ci si trovi proprio in presenza di quei tratti linguistici caricaturali, ad uso esclusivo dei lettori brasiliani, che erano assenti in Mário de Andrade.

Ma l'effetto non è solo comico, ed è tutt'altro che banale sul piano espressivo. Nel contesto del racconto, ognuno degli italianismi "gratuiti" viene infatti a costituire un'affermazione di sé, un orgoglio, un'ostentazione di sicurezza intima, ben più profonda dell'esibizione esteriore di Adriano, il figlio, che si pavoneggia in 
automobile davanti al balcone dell'amata. La lingua imperfetta diventa un ulteriore segno di prestigio, quello dell'origine europea: perfino il quadro del conselheiro, per il semplice fatto di essere bello, viene preso per italiano...

Le frasi-chiave risolutive, "io tenho o capital. O capital sono io", trovano tutta la loro potenza proprio nel fatto che in esse la lingua italiana non costituisce semplicemente un tratto tipizzato, bensì interviene come una presenza assertiva perfettamente adeguata al momento: il pronome in italiano veicola una presa di porere molto più fortemente soggetriva di quanto avrebbe fatto l'equivalente "eu", pronunciato nella lingua dell'interlocutore. $\mathrm{E}$ in questo contesto specifico, l'autoaffermazione ha un valore ancora più ampio. L'imprenditore italiano si impone di fronte al conselheiro brasiliano: non si tratta solo di due personalità, ma di due società a confronto - quella, dinamica e moderna, dell'emigrato che da piccolo commerciante si trasforma in industriale, e quella del possidente che sopravvive in un mondo preindustrializzato e stagnante, in cui la posizione economica è basata più su privilegi ereditati che sull'iniziativa personale. La contrapposizione nettissima è espressa in forma esemplare nella stringata ricostruzione del narratore:

O conselheiro possuía uns terrenos em São Caetano. Coisas de herança. Não lhe davam renda nenhuma. O Cav. Uff. tinha a sua fábrica ao lado.

dove è evidente il divario tra l'improduttività di terreni ricevuti in eredità dal brasiliano e la floridità dell'industria impiantata ex nibilo, proprio li accanto, dall'italiano.

La reiterazione, anzi la recrudescenza, dell'inserto italiano "io", laddove ci si aspetterebbe una autocorrezione linguistica, rafforza ancor di più il carattere performativo delle espressioni italiane. Nella volontà di spiegarsi meglio, invece di emendare formalmente il referenziale "io tenho o capital” con l'equivalente portoghese "eu tenho..." (umile ammissione di estraneità all'ambiente), Melli corregge la sostanza del proprio discorso: lui non soltanto ha ciò che manca al brasiliano (il denaro), ma $\grave{e}$ ciò che il brasiliano non è - un uomo d'affari, nel quale vale la pena investire.

Gli altri italianismi gratuiti ("primo... pense bem", "poi me dê a sua resposta", "domani, dopo domani... quando quiser", "io resto à sua disposiçāo", "dopo... me dá 
a sua resposta", "mi pare... que conhece ele?", "repito un'altra vez: o doutor pense bem") sembrano pronunciati in un contesto altrettanto significativo: come se la condiscendenza e la pazienza dimostrati dall'italiano, che pare voler conquistare il brasiliano convincendolo e non forzandolo, passasse anche attraverso l'offerta di un posto nel proprio sistema di vita, a cominciare dalla lingua materna. Come se, da un lato, insomma, gli mostrasse che la distanza tra loro non è così grande (dato che, tutto sommato, si capiscono pur parlando idiomi diversi), e, dall'altro, lo avesse già incluso nella sua maglia linguistico-culturale al punto da non scomodarsi in inutili sforzi di traduzione.

Lunica resistenza del brasiliano, nel rifiuto secco del titolo di "doutor" - onnipresente titolo di matrice tipicamente italiana, ancor oggi oggetto di satira, e ciononostante qui, ancora una volta, non pienamente caricato di ridicolo - viene dapprima accolta dolcemente dal Melli, il cui intento, dice, è solo facilitar, per poi venire, altrettanto soavemente, del tutto ignorata: per ben altre cinque volte in poche battute, il conselheiro verrà chiamato "doutor". Ma, ormai soggiogato dalla stessa facilità con cui gli è stato presentato l'affare, non sarà più in grado di rifiutare né la lusinga immeritata, né la sua cortese e pragmaticissima motivazione in termini di comodità...

Con la medesima pacifica semplicità con cui il conselheiro accetta l'accorpamento nel sistema dell'italiano, lo stesso narratore accoglierà nel suo testo l'autodefinizione del suo personaggio: "O capital acendeu um charuto [...]. O capital levantou-se" Di nuovo, senza forzature caricaturali: siamo ormai nella sfera del referenziale.

Un ultimo brevissimo caso illustrerà ancora di più il senso di quest'accoglienza: nei Contos de Belazarte, Mário de Andrade dissemina italianismi nella lingua del suo brasilianissimo narratore, come se questi citasse direttamente i personaggi, o ne riprendesse le espressioni. Qualche esempio:

[...] si o nome dêle caía na conversa, a gente só escutava os palavróes que o pai dizia, porca la miserial $^{14}$

14. Mário de Andrade. Contos de Belazarte, op. cit., p. 29. 
Carmela foi contar pro João que ela ia com os três fratelos [... $]^{\text {ss }}$

Chegaram em casa e dito-e-feito: brigaram medonhamente. Porca la miseria, dava mêdo!16

A "figlia dun cane" vinha, emproada porque tinha de seu al pra uns trinta contos, nem sei, e desbaratava com ela por um nadinha. ${ }^{17}$

-Stá zito, guaglion!

Que "stá zito" nada! fome vinha apertando...18

Questa libertà nell'uso dell'italiano instaura un ulteriore contatto tra l'autore e il mondo rappresentato, mentre avvicina, secondo quel movimento che si è descritto all'inizio, questo mondo ai lettori. Il narratore fa propria la lingua dei suoi personaggi, mostrando di averli adottati nel senso più pieno del termine: non solo quindi di averli assunti come oggetti passivi, ma di averli scelti attivamente, in un incontro a doppio senso in cui ognuna della parti entra nella sfera dell'altro.

Se per le espressioni "figlia d'un cane" e "sta' zitto" è chiaro il ricorso alla citazione (il narratore riprende termini e locuzioni direttamente dai personaggi, pur senza preoccuparsi dell'esattezza della dizione), per le altre invece questo uso è più fluido: nel caso di "fratelos" si tratta di una citazione generica, adattata morfologicamente alla lingua del narratore, mentre nei due casi di "porca la miseria", l'esclamazione, sempre prelevata dall'ambiente descritro, viene interiorizzata dal narratore, che la porge ai lettori ( $e$, fra questi, all'autore) mostrando la propria stupefazione in diretta, come fosse ancora impregnato di quell'atmosfera.

Ecco quindi che l'ingresso degli italiani nella narrativa brasiliana mette in gioco un sistema circolare di relazioni tra [narratore-]autore, contesto e pubblico, attivando meccanismi che, a partire da un'unica variabile, quella linguistica, rendono la rappresentazione della fase iniziale dell'immigrazione molto più ricca che una semplice registrazione del fenomeno sociale.

15. Ibid. p. 31.

16. Ibid, p. 56.

17. Ibid, p. 109.

18. Ibid, p. 110. 
Il processo di adattamento degli immigrati al nuovo ambiente non coinvolge solo i protagonisti, ma l'intera società di accoglienza, compresa la sua produzione letteraria.

Allo stesso modo, la "naturalizzazione" degli stranieri nella letteratura brasiliana non si limita a ritrarre i personaggi nel loro sforzo di adattamento progressivo alla lingua e ai modi del nuovo paese, ma, in maniera simmetrica, comporta l'adattamento dei modi e della lingua della narrativa al loro peculiare mondo ibrido. Racconti considerati come cronache, o legati tra loro come capitoli di un romanzo, adottano una lingua plastica che sfrutta le interferenze tra idiomi diversi per creare effetti stilistici sorprendentemente variegati. L'integrazione è avvenuta a doppio senso.

RESUMO: A análise linguïstica dos contos de Mário de Andrade e Alcântara Machado, focados nos imigrados italianos em São Paulo, revela um uso surpreendentemente diverso das interferências do italiano no português, utilizadas como recurso nāo só realístico, mas sobretudo para criar efeitos estilisticos inéditos. PALAVRAS-CHAVE: italiano; português; interferências lingüisticas; Mário de Andrade: Alcântara Machado; estilistica. 